\title{
AVALIAÇÃO DA EFICIÊNCIA DO ATENDIMENTO AMBULATORIAL EM UMA UNIDADE PEDIÁTRICA EM ITABUNA - BA A PARTIR DO ESTUDO DE TEORIA DAS FILAS E SIMULAÇÃO
}

\author{
EVALUATION OF THE OUTPATIENT CARE EFFICIENCY IN A PEDIATRIC UNIT IN \\ ITABUNA - BAHIA FROM THE STUDY OF QUEUEING THEORY AND SIMULATION
}

Jessika Kauanny de Souza Eurico, Sara Meira Moutta², Alex Vinicius Souza Araujo $^{3}$, Francisco Bruno Souza Oliveira ${ }^{4}$

\section{RESUMO}

Filas geralmente são formadas em diversos tipos de serviço. No entanto, quando se trata do setor de saúde, estas esperas podem causar danos e prejuízos que excedem o valor financeiro. Este trabalho tem como objetivo avaliar a eficiência do atendimento em uma unidade pediátrica com atendimento ambulatorial, na cidade de Itabuna, a partir da observação do sistema de atendimento e análise dos dados coletados. Foi utilizado o Simul8 para validar o modelo e identificar como os dados se comportam. Foi verificado que para os intervalos de chegada de usuários e os tempos de atendimento ajustam-se ao tipo de distribuição comum para esse estudo. O modelo é Não Markoviano, pois a distribuição dos intervalos de chegadas é Beta e para o atendimento é uma distribuição Erlang. Além disso, devido o fator de utilização ser maior que 1, notou-se que o sistema é instável, com formação de fila tendendo ao infinito. Por se tratar do atendimento ambulatorial, foram sugeridas algumas propostas de melhoria para o atendimento pediátrico como a distribuição de senhas, instalação de painel informativo e análise para contratação de mais um pediatra, de modo a aumentar 0 nível de serviço no atendimento e redução da fila.

Palavras-chave: Teoria das filas. Atendimento ambulatorial. Simulação. Modelos não Markovianos.

\section{ABSTRACT}

Queues are generally formed in various kinds of service. However, when it comes to the health sector, these delays can cause damages that exceed the financial value. This study aims to evaluate the efficiency of care in a pediatric health unit with outpatient care in the city of Itabuna, from the observation of the service and data analysis system. Simul8 was used to validate the model and to identify how the data behave. It was verified that for the user arrival intervals and the attendance times, they fit the common distribution type for this study. The model is Not Markovian, since the distribution of the arrivals intervals is Beta and for the attendance is an Erlang distribution. Furthermore, because the utilization factor is greater than 1 , it was noted that the system is unstable, with queuing to infinity. Because it's outpatient, was suggested as a proposed improvement to the pediatric care, the distribution of passwords, information panel installation and analysis for hiring more a pediatrician, in order to increase the service level of service and reduce the queue.

Keywords: Theory of Queues. Outpatient care. Simulation. Models Not Markov.
${ }^{1}$ Engenheira de Produção. Universidade Estadual de Santa Cruz.

E-mail:jessika.eurico@gmail.com ${ }^{2}$ Engenheira de Produção, Mestra em Pesquisa Operacional. Universidade Estadual de Santa Cruz.

${ }^{3}$ Engenheiro de Produção, Mestre em Ciência, Inovação e Modelagem em Materiais. Universidade Estadual de Santa Cruz.

${ }^{4}$ Bacharel e Licenciado em Matemática, Mestre e Doutor em Modelagem Computacional. Universidade Estadual de Santa Cruz 


\section{INTRODUCAAO}

O Sistema Público de Saúde apresenta irregularidades no seu funcionamento, ocasionando na geração de filas que podem tanto ser prejudiciais para o paciente, quanto desconfortável. De acordo com o Instituto de Pesquisa Econômica Aplicada - Ipea (IPEA, 2006) essa espera faz parte da rotina dos pacientes que fazem uso do sistema.

A formação de filas ocorre em diversas situações do dia-a-dia em serviços envolvendo atendimento. As filas são vistas, geralmente pelos clientes, como ineficiência do sistema de serviço, causando muitas vezes prejuízos. "A existência de filas e o tempo de permanência nelas estão relacionados com a eficiência do sistema prestador de serviços." (FRAGA, 2014).

Quando se fala em direito à saúde e dever do Estado é importante lembrar que esse direito é integral, de modo que o cidadão tenha acesso ao atendimento e que este atendimento prestado pelo Estado seja feito com qualidade, de modo rápido e eficiente. No entanto a realidade nacional, constantemente vivida no âmbito do Sistema Único de Saúde, é repleta de falhas e dificuldades, são horas de espera para um atendimento que muitas vezes é ineficaz. (LARA FILHO, SILVA; 2012)

A Pesquisa Operacional, por meio da Teoria de Filas, é utilizada para estudo de processos formadores de filas, a fim de produzir indicadores de desempenho do processo real e de cenários propostos, que auxiliarão na tomada de decisão para minimizar o tamanho das filas e a permanência de indivíduos nelas. (FRAGA, 2012)

De acordo com (CARRIÓN, 2007) a teoria das filas tem como objetivo a otimização do desempenho de um sistema. Dessa forma, para alcançar este objetivo, o autor descreve que é necessário analisar os resultados gerados por fórmulas apropriadas a um modelo específico.

O presente trabalho foi realizado a partir da observação do funcionamento e coleta de dados em uma unidade básica de saúde que atende crianças principalmente em casos ambulatoriais, podendo haver internação nos casos mais graves, na cidade de Itabuna BA que atende crianças pelo SUS - Sistema Público de Saúde. O horário de funcionamento é das $08 \mathrm{~h} 00 \mathrm{~min}$ às $18 \mathrm{~h} 00 \mathrm{~min}$, somando um total de cinco mil atendimentos por mês, uma média de mil e duzentas crianças por semana.

O tipo de atendimento realizado nesta unidade é ambulatorial, ou seja, os pacientes chegam à unidade e após cadastro na recepção, aguardam o atendimento do pediatra. Foram realizadas coletas, por meio da observação, em seis dias e horários distintos, ao 
qual se considerou os intervalos de chegadas dos pacientes, a quantidade de pacientes e a duração da consulta (intervalo de atendimento).

A partir dos dados coletados, utilizando o software SIMUL8, foi realizada a modelagem desse sistema para posterior análise da eficiência do atendimento ambulatorial nesta unidade que atende crianças, com a disciplina de filas FIFO, first in- first out, ou seja, o primeiro a chegar é o primeiro a sair do sistema.

\section{MATERIAIS E METODOS}

Este estudo foi realizado para quantificar e classificar o nível de serviço do funcionamento e atendimento de uma unidade básica de saúde na cidade de Itabuna (BA), por meio do estudo e aplicação da Teoria das Filas.

Modelos de filas utilizam probabilidade e modelos estocásticos para analisar filas de espera, e a simulação estima as medições de desempenho reproduzindo o comportamento do sistema real (TAHA, 2008). Foram cronometrados os intervalos de chegadas dos pacientes e os intervalos de atendimento na unidade básica de saúde que funciona basicamente entrada - recepção para cadastro dos pacientes - atendimento com o pediatra. Para o desenvolvimento deste trabalho foi necessário determinar etapas a serem cumpridas para alcançar os objetivos do estudo proposto.

Para realizar a coleta de dados na unidade básica de saúde foi necessária a formulação de uma tabela contendo: número de pacientes, horário de chegada, tempo de atendimento e intervalo entre as chegadas dos pacientes. Os usuários chegam ao sistema pela entrada da unidade básica e aguardam o atendimento na fila. $\mathrm{Na}$ recepção, o atendimento consiste no preenchimento de uma ficha com os dados do paciente e em seguida aguarda a vez de ser atendido pelo pediatra. A disciplina da fila observada é padrão FIFO, ou seja, o atendimento ocorre de acordo com a ordem de chegada à fila.

Após a coleta, os dados foram tabulados e receberam tratamento estatístico para identificação dos outliers, em seguida foram aplicados os testes Anderson Darling e $P$-value para determinar a melhor distribuição que se ajusta aos intervalos de chegadas e atendimento dos pacientes.

Por fim, a partir da análise dos processos de chegada e de atendimento dos clientes, serão aplicadas as equações referentes às Teorias de Filas, de forma a se obter as medidas de desempenho da unidade de saúde. Diante do cenário encontrado na unidade básica de 
saúde ao fim do estudo, serão propostas melhorias para o sistema, visando reduzir o tempo de espera nas filas.

\section{RESULTADOS E DISCUSSÁO}

\subsection{Coleta de dados}

A coleta dos dados foi realizada em seis dias diferentes, considerando o horário que o pediatra começa o atendimento que se inicia às 08h00min da manhã, com pausa de uma hora para o almoço e retorna o atendimento às $14 \mathrm{~h} 00 \mathrm{~min}$, considerando um dia de trabalho de oito horas.

Deste modo, foi utilizado o Critério de Padronização a partir do cálculo da distância entre os quartis e o desvio padrão, como pode ser visto na Figura 1. Assim, o limite superior dessa amostra é 817,62 e o limite inferior $-327,37$. Como não foram adotados valores negativos nesse estudo, considerou-se apenas o limite superior, ou seja, os dados maiores que 817,62 são outliers. E, portanto, foram retirados sete valores dos dados amostrais para não prejudicar a análise do teste de aderência para escolha da distribuição.

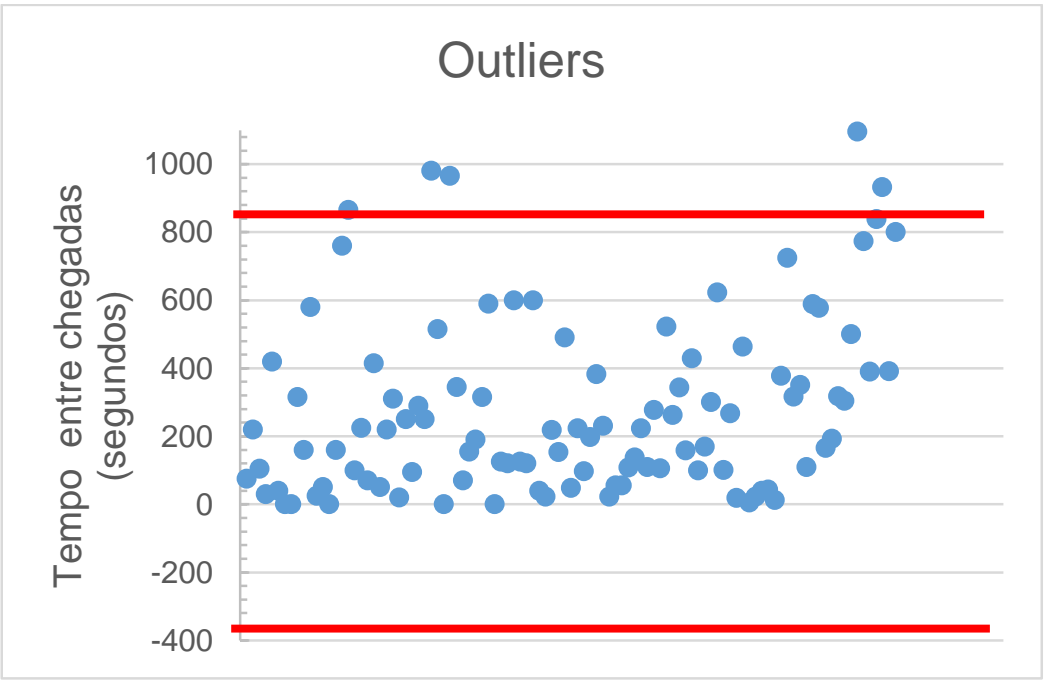

Figura 1. Outliers para os dados de intervalo entre chegadas

A partir do software de ajuste de dados (Statfit) foi gerado o gráfico de distribuição para os intervalos de chegadas com análises estatísticas, como pode ser visto na Figura 2.

A distribuição que melhor se ajustou quanto ao nível de significância foi a distribuição Beta, pois comparada com as outras distribuições apresentadas pelo software, conforme Figuras 3 e 4, esta é a distribuição que melhor se ajusta para descrever os dados. 


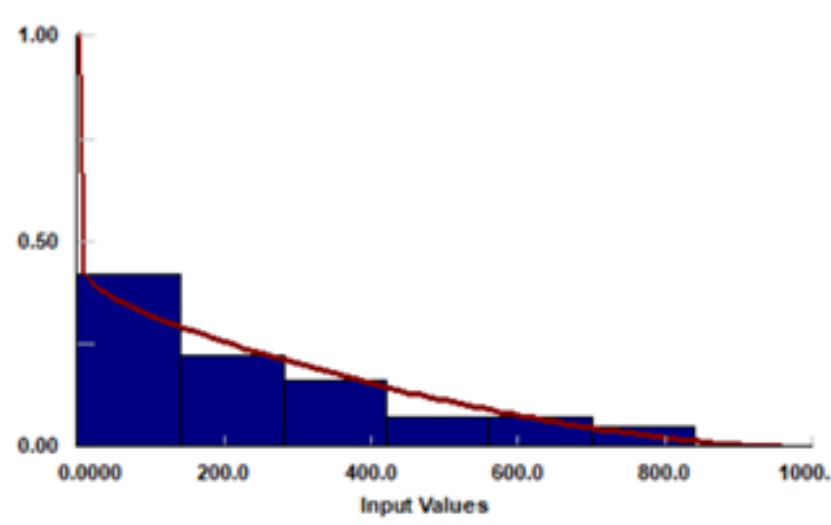

Figura 2. Gráfico de Probabilidade de Intervalo de Chegadas

Auto::Fit of Distributions

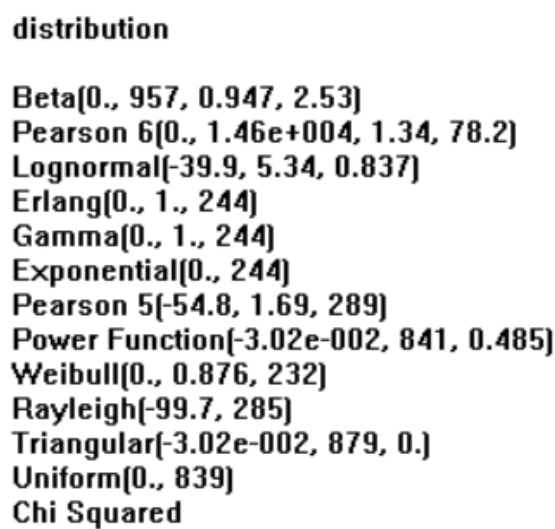

$\begin{array}{ll}\text { rank } & \text { acceptance } \\ 60.6 & \text { do not reject } \\ 45.8 & \text { do not reject } \\ 40.2 & \text { do not reject } \\ 31.7 & \text { do not reject } \\ 31.7 & \text { do not reject } \\ 31.7 & \text { do not reject } \\ 11.8 & \text { do not reject } \\ 2.81 & \text { do not reject } \\ 1.76 & \text { do not reject } \\ 0.211 & \text { reject } \\ 6.08 \mathrm{e}-003 & \text { reject } \\ 0 . & \text { reject } \\ \text { no fit } & \text { reject }\end{array}$

Figura 3. Distribuições avaliadas para dados de intervalo entre chegadas

$\begin{array}{ll}\text { Beta } & \\ \text { minimum }= & 0 . \\ \text { maximum }= & 957.215 \\ \text { p }= & 0.947338 \\ \text { Kolmogorov-Smirnoy } & 2.5286 \\ \text { data points } & \\ \text { ks stat } & \\ \text { alpha } & \\ \text { ks stat[98,5.e-002] } & \\ \text { p-value } & \\ \text { result } & \\ \text { Anderson-Darling } & \\ \text { data points } & \\ \text { ad stat } & \\ \text { alpha } & \\ \text { ad stat[5.e-002] } & \\ \text { p-value } & \\ \text { result }\end{array}$

$\mathbf{9 8}$
$8.75 \mathrm{e}-002$
$5 . e-002$
0.135
0.418
DO NOT REJECT

$\mathbf{9 3}$
$\mathbf{0 . 3 5 7}$
$\mathbf{5 . e - 0 0 2}$
2.49
0.89
DO NOT REJECT

Figura 4. Resultado da análise da distribuição Beta para intervalo entre chegadas. 
Os outliers para os tempos de atendimento também foram identificados de forma análoga ao realizado para os intervalos de chegadas, como pode ser visto na Figura 5.

Tem-se que o limite superior dessa amostra é 628,75 e o limite inferior -45,25. Como não são utilizados valores negativos nesse estudo, será considerado apenas o limite superior, ou seja, os dados maiores que 628,75 são outliers. Foi retirado apenas um dado amostrais para não prejudicar a análise do teste de aderência para escolha da distribuição.

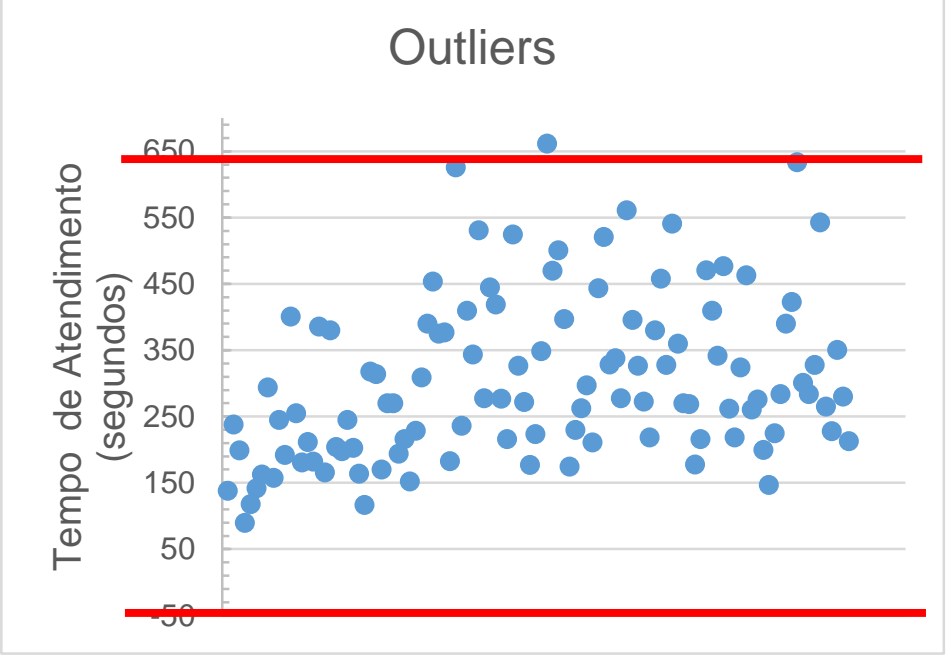

Figura 5. Análise de outliers para os tempos de atendimento

Novamente utilizou-se o software de ajuste de dados (fitting) para gerar o gráfico de distribuição para os intervalos de atendimento, como pode ser visto na Figura 6.

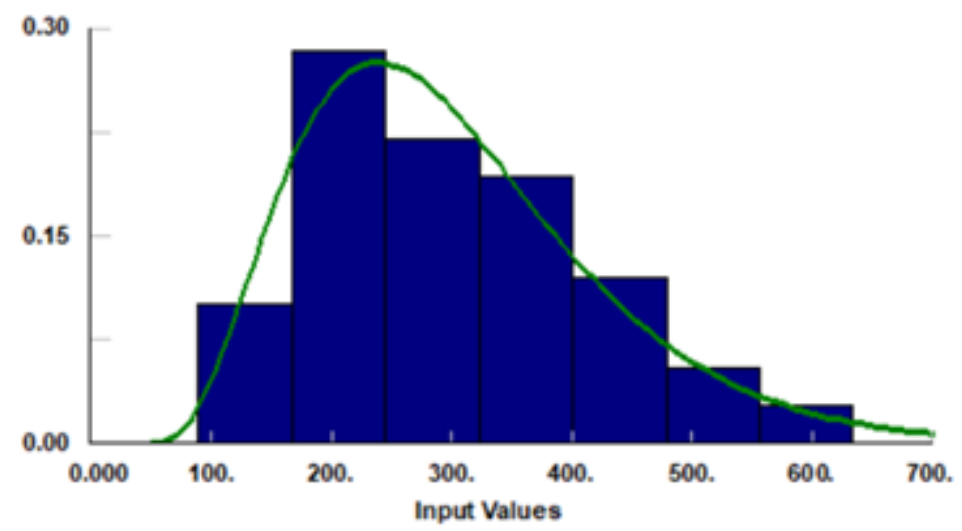

Figura 6. Gráficos Probabilísticos Intervalo de Atendimento

Portanto, para os intervalos de atendimento, após todos os tratamentos estatísticos e execução de testes de aderência de dados apresentados nas Figuras 7 e 8, determinouse a distribuição Erlang para representação dos tempos de atendimento. 


\section{Auto::Fit of Distributions}

distribution

Erlang[50.5, 4., 63.]

Gamma[50.5, 4.42, 56.9]

Lognormal[-35.6, 5.76, 0.349]

Pearson 6[90., 689, 3.91, 13.5]

Pearson 5[-67.5, 10.3, 3.46e+003]

Weibull[81.3, 1.96, 250]

Rayleigh $(79.9,178]$

Triangular[78.8, 654, 202]

Beta[90., 724, 2.04, 3.9]

Uniform[90., 633]

Exponential[90., 213]

Power Function[88.5, 633, 0.883]

Chi Squared

$\begin{array}{ll}\text { rank } & \text { acceptance } \\ \mathbf{1 0 0} & \text { do not reject } \\ \mathbf{9 5 . 5} & \text { do not reject } \\ \mathbf{9 2 . 5} & \text { do not reject } \\ \mathbf{8 8 . 4} & \text { do not reject } \\ \mathbf{8 3 . 1} & \text { do not reject } \\ \mathbf{6 9 .} & \text { do not reject } \\ \mathbf{5 9 . 6} & \text { do not reject } \\ \mathbf{3 5 .} & \text { do not reject } \\ \mathbf{2 7 . 2} & \text { do not reject } \\ \mathbf{0 .} & \text { reject } \\ \mathbf{0 .} & \text { reject } \\ \mathbf{0 .} & \text { reject } \\ \text { no fit } & \text { reject }\end{array}$

Figura 7. Distribuições avaliadas para dados de tempo de atendimento

\begin{tabular}{ll}
\hline 50.5477 & \\
4. & \\
62.9939 & \\
& \\
& 109 \\
& $4.34 \mathrm{e}-002$ \\
& $5 . \mathrm{e}-002$ \\
& 0.128 \\
& 0.982 \\
& DO NOT REJECT \\
& 109 \\
& 0.221 \\
& $5 . \mathrm{e}-002$ \\
& 2.49 \\
& 0.984 \\
& DO NOT REJECT
\end{tabular}

Figura 8. Resultado da análise da distribuição Erlang para tempo de atendimento

\subsection{VALIDAÇAO DO SISTEMA}

Diante da análise dos dados e da distribuição apresentada, o modelo foi validado por meio da utilização do software de simulação, Simul8. Desse modo, constatamos que o sistema apresenta fila. Como pode ser visto na Figura 9, abaixo:

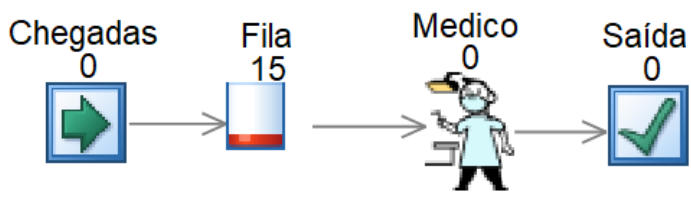

Figura 9. Modelo de simulação pelo software Simul8. 
O simulador apresenta como dados para o sistema atual o número médio de 109,91 pacientes por dia, no horário estudado, tempo médio de permanência no sistema de 6178,73 segundos (aproximadamente 103 minutos) e um percentual de tempo trabalhado para o médico de $100 \%$.

Tabela 1. Resultados do modelo de simulação da situação atual.

\begin{tabular}{cc}
\hline Métrica Avaliada & Resultado \\
\hline Número de Clientes que entraram no sistema & 109,91 \\
Número de Clientes Atendidos & 94,44 \\
Tempo médio no sistema (segundos) & 6178,73 \\
Ocupação (\%) & 100,00 \\
Tamanho Médio de Fila & 21,69 \\
\hline
\end{tabular}

Esses dados apontam para uma sobrecarga do sistema, podendo gerar problemas diversos, desde a demora no atendimento de um caso mais grave até falha em algum diagnóstico em decorrência da fadiga. Na posse desses dados, faz-se necessário estudar alternativas para solução dessa sobrecarga.

\subsection{CRIAÇAO DE CENÁRIO}

Como visto no tópico 3.2, o sistema está sobrecarregado, assim o modelo utilizado será alterado, hipoteticamente, para mais de um servidor de forma análoga ao trabalho desempenhado por (FRAGA, 2012).

Seguindo a método descrito por (FRAGA, 2012), no entanto, considerando apenas o fator de utilização do sistema, temos a mesma taxa de chegada e de atendimento, veja a equação 7, ao qual c representa o número de servidores:

$$
\rho=\frac{\lambda}{c \mu}
$$

Foi avaliado o que ocorre com sistema ao aumentar gradualmente o número de servidores, de forma hipotética.

Para $\mathrm{c}=2$ :

$$
\rho=\frac{0,0040}{2 * 0,0034}=0,58
$$

Quando a capacidade de atendimento é aumentada, para dois servidores, o fator de utilização do sistema é reduzido para 0,58 , ou seja, em $58 \%$ do tempo cada servidor está ocupado, com ociosidade em $42 \%$ do tempo. 
Dessa forma foi observado que se a prefeitura de Itabuna autorizasse a contratação de mais um pediatra, para atender no horário comercial, reduziria o tamanho da fila, pois quanto menor o valor de $\rho$, mais estável é a fila.

Outro ponto vantajoso para essa alternativa é a capacidade de atendimento que a unidade passaria a ter no caso de um aumento repentino da demanda, como por exemplo uma virose ou uma epidemia. Há margem para atendimento de volumes até 50\% maiores, permitindo flexibilidade à unidade.

Além disso, notou-se que não há necessidade de mais de dois servidores no sistema, durante todo o horário de funcionamento, pois este se tornaria ocioso, o que também não é o objetivo, pois além de gerar custos para o município, aumentaria muito a ociosidade do sistema.

\section{CONSIDERAÇOEES FINAIS}

Diante da observação do cenário real do funcionamento da unidade básica de saúde, comparando com as análises estatísticas e o modelo de Teoria das Filas, observamos que o sistema é ineficiente, pois o fator de utilização do sistema é muito elevado, o que indica que a fila tende a infinito.

$\mathrm{Na}$ criação do cenário, foi testado como funcionaria o sistema se fosse aumentado o número de servidores, notamos que com dois servidores o sistema já ficaria ocioso em $42 \%$ do tempo. Uma alternativa seria realizar novos estudos para avaliar os horários de pico e o segundo médico atenderia num horário diferenciado, possivelmente reduzido, diminuindo, desta forma, os gastos com a contratação de um novo médico e também diminuindo o tempo ocioso destes médicos.

A ineficiência do sistema consiste também em o paciente ficar ocioso esperando o seu atendimento. Observamos que no local há uma televisão, porém pouco atrativa para o público alvo. Sugere-se também que seja criado um ambiente agradável para que as crianças se sintam mais confortáveis.

Uma alternativa mais simples e menos dispendiosa seria que fosse estabelecido um número de fichas para atendimento diário, de modo a controlar o crescimento da fila. Além disso, sugere-se a implantação de um painel informativo quanto ao número de senhas disponíveis, posicionamento do atendimento e que seja deixado claro o horário que o médico inicia o atendimento e suas pausas. 
Por fim, apesar das dificuldades encontradas, foi possível uma melhor compreensão da eficiência do atendimento nesta unidade de saúde, o que apontou um resultado negativo. Deste modo, o ideal seria que as autoridades responsáveis avaliassem as possibilidades de melhoria sugeridas neste trabalho de modo a ter um sistema eficiente e estável, o que pode influenciar diretamente na qualidade do serviço prestado às crianças que utilizam este serviço.

\section{REFERÉNCIAS}

BRUNS, Rafael de; SONCIM, Sérgio Pacífico; SINAY, Maria Cristina Fogliatti de. Pesquisa Operacional: Uma Aplicação da Teoria das Filas a um Sistema de Atendimento. In: XXI Encontro Nacional de Engenharia de Produção, XXI ENEGEP, Salvador. Anais... Salvador, 2001.

CHAVES, Ana Laura de Figueiredo; FIGUEIREDO, Ciro José Jardim; VASCONCELOS, Luciano Pacheco; SERRA, Claudio Mauro Vieira. Estudo da Teoria das Filas em um sistema médico-hospitalar na cidade de Belém-PA. IN: XXXI Encontro Nacional de Engenharia de Produção, XXXI ENEGEP, Belo Horizonte. Anais... Belo Horizonte, 2011.

CUYABANO, Beatriz Castro Dias; JUNG, Karen Maria. Teoria de Filas - Processos Estocásticos. Universidade Estadual de Campinas, Campinas (SP), 2009.

DOILE, Luiz Fernando Pacheco Teoria de Filas- Analisando o Fluxo de Atendimento e o Número de Atendentes em um Supermercado. Universidade Federal do Rio Grande do Sul, Porto Alegre (RS), 2010.

GONÇALVES, A. A. Modelo de simulação aplicado na gestão de serviços de saúde. IN: XXV Encontro Nacional de Engenharia de Produção, XXV ENEGEP, Porto Alegre. Anais... Porto Alegre, 2005.

LARA FILHO, Maurílio José, SILVA, Natan Rodrigues da. Otimização do Atendimento ao Público: Estudo de Caso 18 ${ }^{a}$ Regional de Saúde de Cornélio Procópio. Universidade Estadual do Norte do Paraná. Paraná, 2012. 
FONSECA, Felipe Duarte Silva, Uso de Técnicas de Simulação para Otimização da Capacidade de Atendimento do Aeroporto de Ilhéus. Universidade Estadual de Santa Cruz, llhéus, 2016.

FRAGA, Karina Pedrini. Análise de um Processo de Atendimento de Emergência Usando Teoria de Filas. Universidade Estadual do Espírito Santo, São Mateus, 2012.

FROST, J. How to Identify the Distribution of Your Data Using Minitab. The Minitab Blog. Acesso em: 22/06/2016. Disponível em :http://blog.minitab.com/blog/adventures-instatistics/how-to-identify-the-distribution-of-your-data-using-minitab.

IPEA. em: http://www.ipea.gov.br/desafios/index.php?option=com_content\&view=article\&id=9 91 :reportagens-materias\&Itemid=39. Acessado em 15/07/2016

LAW, A. M. Simulation Modeling and Analysis. 5 ed. International Edition. Tucson, Arizona. 2015. apud FONSECA, Felipe Duarte Silva, Uso de Técnicas de Simulação para Otimização da Capacidade de Atendimento do Aeroporto de Ilhéus. Universidade Estadual de Santa Cruz, Ilhéus (BA), 2016.

MENDONÇA, Ednário Barbosa de. Teoria de Filas Markovianas e Aplicações. Universidade Estadual da Paraíba, Campina Grande, 2014.

MORAES, Flávio Gomes de; SILVA, Gecirlei Francisco da. REZENDE, Tacyanne Assis. Introdução à Teoria das Filas. Universidade Federal do Mato Grosso, Cuiabá, 2011.

TAHA, Hamdy A. Pesquisa Operacional. 8 ed. São Paulo: Pearson Prentice Hall, 2008.p. 366. 